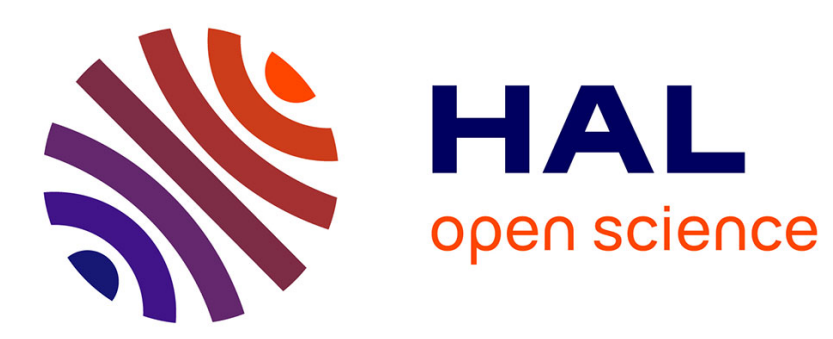

\title{
L'Histoire naturelle de la religion et les Dialogues sur la religion naturelle
}

\author{
Alexandre Simon
}

\section{To cite this version:}

Alexandre Simon. L'Histoire naturelle de la religion et les Dialogues sur la religion naturelle. Philosophique, 2009, 848, pp. 93-122. hal-00448859

\section{HAL Id: hal-00448859 \\ https://hal.science/hal-00448859}

Submitted on 4 Feb 2010

HAL is a multi-disciplinary open access archive for the deposit and dissemination of scientific research documents, whether they are published or not. The documents may come from teaching and research institutions in France or abroad, or from public or private research centers.
L'archive ouverte pluridisciplinaire HAL, est destinée au dépôt et à la diffusion de documents scientifiques de niveau recherche, publiés ou non, émanant des établissements d'enseignement et de recherche français ou étrangers, des laboratoires publics ou privés. 


\section{L'histoire naturelle de la religion et les Dialogues sur la religion naturelle}

Les deux principaux écrits de Hume sur la religion sont L'histoire naturelle de la religion, publiée en 1757 dans Four Dissertations, et les Dialogues sur la religion naturelle, publiés après la mort de leur auteur, en $1779^{1}$. Si l'on s'en tient à la distinction qu'établit l'Introduction de L'histoire naturelle entre la question du fondement de la religion dans la raison et celle de son origine dans la nature humaine, il est tentant d'attribuer à chacun de ces deux textes un objet propre, déterminant un type de discours philosophique particulier. L'histoire naturelle, comme son titre l'indique, développerait un discours causal, principalement généalogique, sur les religions positives, toutes populaires en leur fond, tandis que les Dialogues auraient à charge de mener l'examen critique des prétentions philosophiques de la religion naturelle ${ }^{2}$. Cette distinction est inévitable. Si la religion en général commence avec la croyance en l'existence d'une puissance surnaturelle invisible - ou de plusieurs -, il faut cependant reconnaitre qu'il y a bien deux principales sortes de religion. Dans la première, l'homme ignore les mécanismes du monde naturel, parce qu'il se rapporte à la nature dans un but immédiatement pratique, celui de satisfaire ses besoins vitaux. Dans la seconde au contraire, l'homme se rapporte à la nature dans un but principalement théorique, celui d'en connaître l'ordre, et d'en inférer l'existence d'un créateur ou d'un auteur intelligent. En somme il y aurait d'un côté la religion du peuple, ou de l'humanité encore inculte, et de l'autre la religion des savants, ou de l'humanité civilisée. Mais les choses ne sont pas si simples, ni à l'intérieur de la religion, ni dans son rapport avec la philosophie, ni par conséquent dans le rapport entre L'histoire naturelle et les

\footnotetext{
1. Sur l'histoire complexe de la publication de chacune de ces deux œuvres, voir pour L'histoire naturelle E.C. Mossner, «Hume's Four Dissertations : An essay in biography and bibliography » in Modern Philology Review, XLVIII, 1, 1950, p. 37-57 ; et pour les Dialogues l'appendice M de l'édition des lettres de Hume par J.Y. Greig, The letters of David Hume, Oxford, Clarendon Press, 1932, vol. II, p. 453-454 et l'Introduction de M. Malherbe à sa traduction des Dialogues sur la religion naturelle, Paris, Vrin, 1997, p. 11-13. C'est cette traduction qui sera citée ici, le titre abrégé (DRN) étant suivi de l'indication de la partie en chiffres romains et de la page en chiffres arabes.

2. Voir par exemple A. Leroy, La critique et la religion chez David Hume, Paris, Alcan, 1929, p. $246-248$; J.C.A. Gaskin, Hume's Philosophy of Religion, London, The Macmillan Press, 1978, 1, ii.
} 
Dialogues. Car d'une part, en montrant que le polythéisme, religion primitive de l'humanité inculte, conduit naturellement au théisme, L'histoire naturelle montre par là même que le théisme, dans lequel il faut comprendre la religion naturelle elle-même, a ses origines dans la superstition populaire. D'autre part, les trois dernières parties des Dialogues, consacrées aux attributs moraux de la divinité (parties X et XI) et à l'utilité ou la nécessité de la religion pour la morale et la société (partie XII), mettent en évidence les prétentions pratiques, et non simplement spéculatives, de la religion naturelle. Autrement dit, la superstition populaire a un devenir philosophique ou spéculatif, dans le théisme, et inversement la religion naturelle rejoint les religions positives dans leur prétention à régir la conduite humaine. C'est pourquoi, loin d'être totalement distincts et indépendants, le discours de L'histoire naturelle et celui des Dialogues se croisent et sont dans un rapport d'étroite intertextualité ${ }^{3}$. Plus précisément, une lecture interne de L'histoire naturelle suffit tout d'abord pour comprendre que la religion naturelle est bien, au même titre que les autres sortes de religion, l'objet de cette dissertation. Mais ainsi comprise, L'histoire naturelle porte alors un éclairage sur les Dialogues, en ce qu'elle permet de montrer que le ressort de l'argumentation de Cléanthe comme de Déméa dans les Dialogues est la superstition.

Dans quelle mesure la religion naturelle est-elle l'objet de L'histoire naturelle de la religion? Si l'on en croit l'Introduction de cette dissertation, elle ne l'est pas du tout, puisque Hume y circonscrit précisément son objet en excluant la religion naturelle de la recherche causale qui va suivre : «Toute enquête qui porte sur la religion étant de la plus haute importance, deux questions retiennent plus particulièrement notre attention, celle qui concerne son fondement dans la raison et celle qui concerne son origine dans la nature humaine. Heureusement la première question, qui est la plus importante, admet la plus évidente ou du moins la plus claire des solutions. L'entier

3. M. Malherbe a montré que la distinction entre la religion savante et la religion populaire ne recouvre pas exactement la distinction entre la question du fondement et celle de l'origine de la religion (op. cit., p. 16-25). 
agencement de la nature annonce un auteur intelligent; et tous ceux qui se livrent à une enquête rationnelle ne peuvent, après une sérieuse réflexion, suspendre un instant leur croyance quant aux premiers principes du pur Théisme et de la pure Religion. Mais l'autre question, celle qui concerne l'origine de la religion dans la nature humaine, est sujette à plus de difficultés ${ }^{4}$. Dans le corps de la dissertation, Hume accusera cette distinction en opposant à plusieurs reprises, comme il l'avait fait en 1741 dans l'essai «Superstition et enthousiasme », « la vraie religion » à ce qu'il appelle ses « corruptions » ${ }^{5}$. Dans L'histoire naturelle, la vraie religion est assimilée au pur théisme, c'est-à-dire au théisme exempt de toute superstition, parce qu'il repose sur une connaissance de la nature, tandis que la superstition est une crainte jointe à l'ignorance des mécanismes du monde naturel. L'histoire naturelle de la religion étant tout entière l'histoire de la superstition, le théisme dont il est question dans cet écrit comme ayant son origine dans le polythéisme n'est donc pas celui des philosophes. C'est le monothéisme populaire sur lequel les prêtres assoient leur pouvoir, c'est-à-dire principalement le théisme chrétien, comme religion prétendument révélée. La religion naturelle est donc entièrement extérieure à l'objet propre de L'histoire naturelle.

Mais la contrepartie de cette interprétation est difficile à admettre. En laissant de côté la religion naturelle, L'histoire naturelle de la religion serait incomplète, puisqu'elle ne rendrait compte que de la plupart des religions, mais non de toute espèce de religion, et usurperait donc son titre d'histoire naturelle de la religion. Ce qui lui échapperait, c'est ce qu'il y a de spécifiquement religieux dans le pur théisme, que Hume appelle aussi la pure religion. Est-ce à dire que la religion vraie n'est rien qu'$^{\prime}$ une espèce de philosophie ${ }^{6}$ ? Mais alors, pourquoi continuer de parler de religion?

A partir de là, on peut avancer deux hypothèses concurrentes : ou bien il faut admettre que L'histoire naturelle de la religion est incomplète, pour les raisons qui viennent d'être évoquées, ou

\footnotetext{
4. L'histoire naturelle de la religion et autres essais sur la religions, Paris, Vrin, 1996, trad. M. Malherbe, p. 39 (noté ciaprès HNR, suivi de l'indication de la section de L'histoire naturelle en chiffes romains - ou de la mention du titre complet de l'un des autres essais contenus dans ce recueil - et de la page en chiffres arabes).

5. HNR, « Superstition et enthousiasme », 33 ; IX, $74 ;$ X, 75.
} 
bien il faut voir dans le discours théiste que Hume tient dans L'histoire naturelle une concession rhétorique destinée à dissimuler provisoirement l'objet ultime de cet écrit, la religion naturelle, derrière une apparente adhésion aux conclusions de celle-ci. Comment étayer cette dernière interprétation?

Elle implique tout d'abord que, de l'avis de Hume, la religion naturelle n'est pas, en réalité, une religion vraie ${ }^{7}$. Pour s'en convaincre, il n'est pas nécessaire de se rapporter à la critique sceptique des Dialogues, il suffit de lire le corollaire général qui conclut L'histoire naturelle, lequel renverse totalement la profession de foi théiste de l'Introduction. Certes, le premier paragraphe du corollaire répète cette profession de foi, mais la suite du texte avance une description pessimiste du monde à laquelle Philon aura recours dans la dixième partie des Dialogues pour réfuter l'inférence des attributs moraux de la divinité, mais que tout lecteur de L'histoire naturelle, averti des réquisits empiriques du théisme expérimental, ne manquera pas de tenir pour un argument dirigé contre cette même inférence, avant même de lire les Dialogues : «Le tout est un abîme, une énigme, un mystère inexplicable. Le doute, l'incertitude, la suspension du jugement semblent les seuls résultats de notre examen le plus attentif sur ce sujet $»^{8}$. Et le développement qui aboutit à cette conclusion sceptique est particulièrement habile, puisqu'il commence par admettre l'existence d'une religion vraie, pour finir par en ruiner les fondements, et cela en rendant les autres religions responsables du mal moral dans le monde : supposé que le théisme soit vrai et bénéfique, les inconvénients des autres religions sont tels qu'ils font régner le plus grand désordre dans les affaires humaines, désordre qui rend alors plus que douteuse l'existence d'un sage auteur de la nature ! C'est ce renversement qui structure l'ensemble du texte du corollaire, lequel s'ouvre sur une profession de foi théiste pour se refermer

\section{DRN, XI, 221.}

7. La fin de l'essai «De l'immortalité de l'âme », ainsi que celle des Dialogues (par la bouche de Philon), semblent attendre de la religion révélée une réponse aux questions qui dépassent la pure raison (HNR, «De l'immortalité de l'âme », 114 ; DRN, XII, 227). Mais il ne peut s'agir que d'artifices rhétoriques de la part de Hume, puisque la section X de l'Enquête sur l'entendement humain, consacrée aux miracles, laisse clairement comprendre que Hume n'adhère à aucune religion révélée. Et la réduction naturaliste de L'histoire naturelle le confirmera. Ce qui importe ici, c'est de montrer que la religion naturelle n'est pas non plus, à ses yeux, la religion vraie, et que par conséquent il n'y a pas pour Hume de religion vraie du tout.

8. HNR, XV, 104. 
sur une profession de foi sceptique. Mais cette contradiction entre la reconnaissance d'une religion pure et le constat des inconvénients de la religion structure presque chacun des paragraphes du texte, et lui imprime ainsi un rythme inconfortable. Le paragraphe suivant est le plus explicite : «C'est un noble privilège, pour la raison humaine, de s'élever à la connaissance de l'Être suprême et de pouvoir inférer, à partir des a uvres visibles de la nature, un principe aussi sublime que son Créateur suprême ; mais voyez le revers de la médaille. Observez la plupart des nations et la plupart des époques. Examinez les principes religieux qui se sont en fait imposés dans le monde. Vous vous persuaderez avec peine qu'ils sont autre chose que les rêves d'un homme malade. Ou peut-être les considérerez-vous comme les jeux et les fantaisies d'un singe vêtu d'une forme humaine, plutôt que comme les affirmations sérieuses, positives et dogmatiques de celui qui se glorifie du titre d'être rationnel » ${ }^{9}$. Or en plaçant ce développement sceptique en conclusion de la dissertation, Hume lui donne bien davantage de crédit qu'au discours théiste qu'il avait tenu occasionnellement dans le reste du texte.

A la lumière du final sceptique de L'histoire naturelle, ce discours théiste n'apparaît plus que comme une concession rhétorique provisoire au lecteur théiste. La fonction de cet artifice ou de cette feinte est de permettre à l'auteur de tranquilliser tout d'abord le lecteur sur la question de la religion naturelle. Cette question, «qui est la plus importante», reçoit «heureusement» une réponse claire ${ }^{10}$. Mais c'est pour mieux attirer son attention sur les autres sortes de religion, et cela dans le but ultime de lui faire comprendre, comme on va le voir, que la religion naturelle en provient ! Amadoué par un théisme de façade jusqu'à la fin de la dissertation ou presque, le lecteur sera d'autant mieux déconcerté par sa conclusion sceptique. Et cette conclusion, si l'on ne veut pas la couper du reste du texte, doit rejaillir sur lui, et commander une relecture, en lecteur avisé, de l'ensemble de la dissertation. Que nous apprend cette deuxième lecture ?

Le premier effet de la conclusion sceptique de L'histoire naturelle, c'est de réinsérer dans le

9. HNR, XV, 103-104. 
champ d'investigation causale de cette histoire la religion naturelle elle-même, qui en avait été exclue par l'artifice rhétorique de l'Introduction. Si même le théisme expérimental n'est pas une religion vraie ou pure, comme invite à le penser le corollaire général, alors L'histoire naturelle de la religion doit être complète, c'est-à-dire que sa réduction généalogique de toute religion à la superstition doit de manière ultime affecter le théisme spéculatif lui-même.

La première section de la dissertation a pour fonction de rendre possible cette réintégration de la religion naturelle dans une histoire naturelle de la religion. Elle constitue comme une seconde introduction à l'ensemble du texte, puisqu'elle ne traite pas encore de la question de l'origine de la religion dans la nature humaine, mais de cette question de fait historique qui est celle de savoir quelle fut la religion originelle ou primitive de l'humanité. En établissant que c'est le polythéisme et non le théisme philosophique, elle donne certes un premier objet ou un point de départ à l'histoire naturelle qui va commencer avec la deuxième section, mais elle prouve en même temps l'historicité de la religion naturelle. Si la religion naturelle n'est pas, en réalité, naturelle, c'est certes au sens où elle n'est pas rationnelle, comme le laissera penser le corollaire général avant que les Dialogues n'en fassent la démonstration complète. Mais c'est aussi, et par conséquent, au sens où elle n'a rien d'originel, comme le croient les théistes lorsqu'ils affirment que tout homme peut en découvrir les vérités par un bon usage de sa raison ${ }^{11}$. La concession rhétorique, véhiculée par la maxime selon laquelle « la corruption des meilleures choses engendre les pires », allait pourtant dans ce sens, en présentant les passions religieuses violentes comme des corruptions de la religion vraie ou pure ${ }^{12}$. Mais L'histoire naturelle dément cette affirmation, en montrant qu'« il doit apparaitre impossible que le théisme ait pu être par raisonnement la première religion de la race humaine, et donner ensuite naissance, par sa corruption, au polythéisme et à toutes les diverses superstitions du monde

10. HNR, Introduction, 39.

11. Voir Locke, Essays on the Law of Nature, II et Essai sur l'entendement humain, Livre I, ch. IV, § 8-16: si l'idée vraie de Dieu n'est pas innée, comme le prouve l'existence des anthropomorphites et des polythéistes, l'esprit humain est néanmoins doté de facultés propres à le conduire à cette connaissance par voie naturelle, facultés dont le mauvais usage qu'en font la plupart des hommes explique la diversité des croyances religieuses.

12. HNR, IX, 74 (note de Hume, qui cite la maxime en latin) et X, 75. C'est par cette même maxime que s'ouvre 
païen $»^{13}$. Un tel retour au polythéisme après l'apparition du théisme est improbable, pour ne pas dire impossible. Par conséquent, le lieu commun théiste qui fait de la religion naturelle une chose bonne et originelle dont la superstition et l'enthousiasme ne seraient que les corruptions n'exprime qu'une illusion rétrospective que devra faire tomber une histoire naturelle de la religion incluant dans son champ d'investigation causale la religion naturelle.

Si donc la religion naturelle est historique, et non originelle, il est bien légitime d'en faire l'histoire. Mais la circonstance qui rendait improbable l'antériorité historique de la religion naturelle par rapport au polythéisme rend en même temps problématique sa dérivation à partir de celui-ci. Si le théisme philosophique n'a pu être la première religion de l'humanité, c'est parce que la contemplation de la nature et l'interrogation sur les causes finales dépassent infiniment les capacités d'une humanité inculte trop préoccupée par ses besoins les plus élémentaires ${ }^{14}$. Comment donc l'humanité a-t-elle pu passer de la religion populaire à la religion philosophique ?

Pour comprendre cette évolution, il faut la décomposer en deux moments. Le polythéisme donne tout d'abord naissance à un théisme populaire, avant que celui-ci, en rencontrant la philosophie naturelle, ne fasse apparaître le théisme proprement philosophique. Mais avant que la section VI de L'histoire naturelle ne montre cela, les sections II à V, consacrées au polythéisme, préludent à cette dérivation.

La deuxième section semble à première vue se borner à expliquer comment l'humanité inculte donne un sens aux contradictions de la nature - au mélange universel des biens et des maux - dans la croyance en l'existence de divinités multiples et inconstantes. Mais sa portée va bien audelà, car elle est habilement composée. Après avoir une nouvelle fois rassuré le lecteur théiste en un premier paragraphe affirmant que l'unité de la nature conduit naturellement à lui attribuer un auteur unique - car il ne faut pas multiplier les causes sans nécessité, Hume décrit longuement dans le paragraphe suivant l'universelle compensation des biens par les maux, physiques ou moraux. Or 
cette description non seulement est proche de la vision du monde sur laquelle s'appuiera Philon dans les parties X et XI des Dialogues pour réfuter les attributs moraux de la divinité, mais elle trouvera déjà un écho dans le corollaire général de L'histoire naturelle. Hume s'y exprimera en ces termes : «Le bien et le mal se mêlent et se confondent universellement ; de même le bonheur et le malheur, la sagesse et la folie, la vertu et le vice. Rien n'est pur ni tout d'une pièce. Une compensation universelle s'impose dans toutes les conditions d'être et d'existence $»^{15}$. Si les hommes scrutaient attentivement les œuvres de la nature, ce n'est pas une théodicée qui devrait sortir de cette observation, solution qui implique «un projet ou une intention unique, quoique inexplicable et incompréhensible » ${ }^{16}$; c'est bien plutôt l'hypothèse manichéenne, amputée de sa supposition polythéiste de deux divinités opposées, selon laquelle le monde est gouverné par une force aveugle, indifférente au bien et au mal ${ }^{17}$. Si donc les contradictions de la nature discréditent le système théiste, c'est que celui-ci ne doit être qu'une manière plus raffinée que le polythéisme de donner un sens à ces contradictions, à défaut de pouvoir les résoudre, sans qu'il y ait par conséquent de véritable solution de continuité entre ces deux sortes de religion.

La troisième section contribue à établir cette continuité, envisagée cette fois-ci du point de vue de l'anthropomorphisme. Son objet premier est de montrer comment l'humanité inculte résout un second problème, celui des causes inconnues des événements heureux mais surtout malheureux de l'existence. Afin de se donner un objet, les passions de l'espoir et de la crainte suscitent la construction imaginaire de cet objet, selon une projection des sentiments humains de malveillance et de bienveillance sur les contradictions de la nature. Mais cette projection polythéiste, dont Hume rappelle la fécondité littéraire, n'est qu'un cas particulier de ce qui est chez les hommes «une tendance universelle à concevoir tous les êtres à leur ressemblance et à transférer à tous les objets

\footnotetext{
14. HNR, II, 46-47.

15. HNR, XV, 102.

16. Ibid. (je souligne).

17. DRN, XI, 202-203. C'est évidemment Philon qui suggère cette hypothèse.
} 
les qualités auxquelles ils sont habitués et familiarisés et dont ils ont une conscience intime ${ }^{18}$. Et Hume de remarquer en passant que « les philosophes eux-mêmes ne peuvent entièrement échapper à cette fragilité naturelle : ils ont souvent accordé à la matière inanimée l'horreur du vide, des sympathies, des antipathies et d'autres affections de la nature humaine ${ }^{19}$. Certes, Hume semble ici viser uniquement les «péripapéticiens », si l'on rapproche ce passage d'un paragraphe du Traité où les anthropomorphismes des philosophes côtoyaient déjà les prosopopées des poètes ${ }^{20}$. Mais le paragraphe correspondant de L'histoire naturelle se clôt sur l'observation qu' «il faut peu de temps pour que nous leur donnions [aux causes inconnues] la pensée, la raison, les passions et parfois même les membres et les formes humaines, afin de les amener à une plus grande ressemblance avec nous-mêmes $»^{21}$. Si l'homme ignorant projette ses craintes et ses espoirs sur les divinités en leur attribuant malveillance et bienveillance, quoi de plus naturel que des philosophes attribuent la pensée et la raison à la puissance invisible dont ils infèrent l'existence à partir de la contemplation de la nature ? Que l'anthropomorphisme soit essentiel au théisme expérimental, les Dialogues le montreront ${ }^{22}$.

La quatrième section, en montrant «que le polythéisme ne considère pas ses divinités comme la cause de la création ou de la formation du monde », traite subsidiairement de la question de savoir ce qui peut recevoir le nom de religion, en toute propriété de langage. Mais ici comme ailleurs, c'est un double langage que tient Hume. La réponse à la question est certes affirmée de manière ferme : le polythéisme mérite à peine qu'on lui applique le nom de religion, qui devrait à proprement parler être réservé au seul théisme. Mais cette affirmation est susceptible d'une double interprétation, l'une théiste, l'autre naturaliste. Le lecteur théiste de L'histoire naturelle se plaira, en lisant cette section, à penser que le polythéisme n'est pas une vraie religion, parce que sa foi s'en

\footnotetext{
18. HNR, III, 48.

19. Ibid.

20. Voir Traité de la nature humaine, Livre I, Partie IV, sect. III (noté ci-après TNH, suivi de l'indication du livre, de la partie et de la section en chiffres romains, puis de la page en chiffres arabes dans la traduction du Livre I (seul cité) par $\mathrm{Ph}$. Baranger et $\mathrm{Ph}$. Saltel parue sous le titre L'entendement, Paris, GF-Flammarion, 1995).

21. HNR, III, 49 (je souligne).
} 
tient au bas de l'échelle des êtres de la théologie médiévale, en limitant sa croyance à l'existence d'êtres analogues aux elfes et aux fées de ce système, à l'exclusion des anges et de Dieu. Si le polythéisme n'est pas véritablement une religion, c'est parce qu'il n'est pas la religion vraie. C'est par ce discours que Hume commence, comme à son habitude dans L'histoire naturelle, par flatter son lecteur théiste. Mais que dit le reste de la section ? Ce qui prouve que le polythéisme ne considère pas ses divinités comme la cause de la création ou de la formation du monde, c'est la manière sacrilège, aux yeux d'un théiste, dont il les traitent dans son culte, dans sa littérature et dans ses cosmogonies. Les Chinois battent leurs idoles, quand leurs prières ne sont pas exaucées ; le grotesque dans lequel les comédies d'Aristophane représentaient les dieux ne choquait ni les Athéniens ni les Romains ; et les Physiologues faisaient communément dériver et les hommes et les dieux du chaos des éléments. Or ces phénomènes fournissent la preuve que les polythéistes craignaient beaucoup moins leurs dieux que les théistes ne craignent le leur lorsqu'ils lui accordent le titre élogieux de créateur du monde. Or la crainte superstitieuse est l'essence même de la religion ${ }^{23}$. C'est pourquoi le polythéisme, moins superstitieux que le théisme, est dans cette mesure moins religieux. Pour l'auteur naturaliste de L'histoire naturelle de la religion, dire que le polythéisme mérite à peine le nom de religion, qu'il faudrait dès lors réserver au théisme, c'est dire qu'il n'est pas véritablement une religion, et que le théisme, à défaut d'être la religion vraie rationnelle - est en revanche la véritable religion, au sens où c'est en lui que se manifeste le plus la spécificité du phénomène naturel de la religion, à savoir la superstition ! En toute propriété de langage naturaliste - et ce langage est le vrai langage de L'histoire naturelle - c'est donc le théisme qui mérite le plus le nom de religion, étant la forme la plus superstitieuse de religion.

On ne peut nier que le polythéisme ait son origine dans la crainte des événements naturels jointe à l'ignorance de leurs causes. Mais ce que montre la cinquième section de L'histoire naturelle,

22. Voir infra, p. 23-27.

23. Si dans ses premiers mouvements l'enthousiasme est irréductible à la superstition, puisqu'il en est l'opposé, il s'éteint rapidement, soit pour laisser place à la philosophie (voir HNR, «Superstition et enthousiasme », 33-38), soit pour tourner en superstition (DRN, XII, 224-225). 
c'est que dans une nation civilisée où persiste le polythéisme, l'élément superstitieux de cette religion laisse place aux passions calmes qui président à la création artistique, par où la religion acquiert une utilité sociale. La tendance du polythéisme à localiser ses divinités dans de multiples objets sensibles, outre qu'elle soulage l'imagination dans son effort pour se représenter les puissances invisibles, donne naissance à deux formes de polythéisme plus raffinées : l'allégorie et le culte des héros. Or ce sont là des inventions conscientes de la partie cultivée des sociétés grecque et romaine, destinées à exercer une influence socialement bénéfique sur le peuple. Par définition, l'allégorie implique la conscience du degré second de son propre discours, et donc du caractère fictif des objets concrets qu'elle utilise pour signifier des abstractions. Or cette médiation place la divinité dans une distance caractéristique de l'objet d'une passion calme et diminue d'autant la violence de la crainte superstitieuse. C'est la première utilité sociale de l'allégorie. Mais en figurant artistiquement les passions et les occupations qui cimentent la société, la fabulation allégorique berce les hommes avec des histoires qui leur racontent la division du travail sur laquelle repose toute société humaine. C'est la seconde utilité sociale de l'allégorie que de renforcer ce fondement de la vie en société. Et le culte des héros remplit une fonction analogue, en imprimant à la mémoire collective le souvenir de grands hommes faisant figure de modèles. Or c'est là un artifice conscient et utile des poètes et des prêtres païens ${ }^{24}$. Cette section, en montrant comment des nations peuvent se civiliser sans l'intervention du théisme, prépare en même temps la comparaison entre le théisme et le polythéisme (sections IX à XIV) qui tournera à l'avantage du second.

Maintenant, comment le théisme a-t-il pu sortir du polythéisme ? Et surtout, cette dérivation concerne-t-elle en quelque manière le théisme philosophique, c'est-à-dire la religion naturelle ?

La section VI répond à ces deux questions. Elle s'ouvre par le rappel de l'historicité de la religion naturelle, c'est-à-dire de son caractère non originel, en dépit de son ancienneté : «La doctrine d'une divinité suprême, auteur de la nature, est très ancienne et elle s'est répandue à travers

24. HNR, V, 60. 
de vastes nations très peuplées, où tous les rangs et toutes les conditions l'ont embrassée. Mais qui penserait qu'elle a dû son succès à la force déterminante des invincibles raisons, sur lesquelles elle est indubitablement fondée, se montrerait peu instruit de l'ignorance et de la stupidité du peuple et de ses préjugés incurables en faveur de ses superstitions particulières. Même aujourd'hui et en Europe, demandez à un homme du peuple pourquoi il croit en un créateur du monde tout puissant ; il ne mentionnera jamais la beauté des causes finales qu'il ignore totalement $[\ldots] »^{25}$. Si donc ce n'est pas en produisant une conviction rationnelle que la religion naturelle s'est imposée à une partie de l'humanité, ce doit être par la force de quelque passion ! On aura pourtant de la peine à le croire, étant donné que l'une des circonstances qui donnèrent naissance à la religion naturelle est l'acquisition d'une connaissance de la nature dont l'absence est toujours responsable de la superstition vulgaire. Certes, mais Hume laisse alors entendre de façon claire que la connaissance de la nature aurait plutôt engendré l'athéisme et l'aurait maintenu, si la persistance de la superstition dans l'esprit humain n'avait entraîné les hommes même les plus savants dans la religion naturelle : «Mais la grande majorité des hommes saisit si peu ces arguments [ceux du théisme expérimental] que, partout où elle voit tous les événements attribués à des causes naturelles et l'interposition d'une divinité écartée, elle a tendance à détecter l'infidélité la plus grossière. Un peu de philosophie, dit Lord Bacon, rend les hommes athées, beaucoup de philosophie les réconcilie avec la religion. Sous l'influence des préjugés superstitieux, l'homme apprend à mettre l'accent là où il ne faut pas. Lorsqu'il perd cet appui et qu'il découvre par un peu de réflexion que le cours de la nature est régulier et uniforme, toute sa foi chancelle et s'effondre. Mais lorsque, par suite d'une plus grande réflexion, il apprend que cette régularité, cette uniformité même sont la plus forte preuve d'un dessein et d'une intelligence suprême, il revient à la croyance qu'il avait abandonnée et il est alors capable de l'établir sur des fondements plus solides et plus durables $»^{26}$. Le théisme philosophique n'est donc pas épuré de toute superstition par l'apport de la philosophie naturelle. Au contraire, il 
manifeste la persistance du religieux à l'intérieur de la philosophie, c'est-à-dire de la superstition au sein de la raison humaine. Ces «fondements plus solides et plus durables » que la philosophie naturelle donne à la croyance religieuse, ce sont les arguments expérimentaux du théisme qui, apparemment affectés d'un certain degré de probabilité, ont quelque apparence philosophique par laquelle ils rendent la superstition elle-même plus crédible. C'est cette persistance de la superstition dans la religion naturelle elle-même qui réintègre pleinement celle-ci dans le champ d'une histoire naturelle de la religion : «Nous pouvons donc former la conclusion générale que, puisque chez les nations qui ont embrassé le théisme, cette doctrine est encore construite sur des principes irrationnels et superstitieux, le commun n'est jamais conduit à cette opinion par voie d'argument, mais par une certaine chaîne d'idées plus conformes à son génie et à ses capacités » ${ }^{27}$. Par conséquent, c'est d'un théisme populaire que le théisme spéculatif doit tirer toute son influence. Il ne reste donc plus qu'à faire dériver le théisme populaire du polythéisme pour montrer que la religion naturelle a son origine dans la superstition.

C'est ce qu'accomplit la suite de la section, en montrant par quelle « chaîne d'idées plus conformes à son génie et à ses capacités » l'homme du commun est conduit au théisme. Cette chaîne d'idées a pour ressort l'opposition entre deux principes superstitieux contradictoires, la tendance à l'idolâtrie ou à l'anthropomorphisme et la tendance à la louange. La naissance du théisme à partir du polythéisme s'amorce tout d'abord par une projection anthropomorphique particulière, celle de la structure hiérarchique de la société politique des hommes : le dieu qui deviendra unique n'est au départ qu'un dieu plus éminent que les autres, et qui se distingue de ceux-ci comme le prince de ses sujets. Puis la frénésie louangeuse prend le relais pour parfaire l'éminence de cette divinité

27. HNR, VI, 64 (je souligne). Il en va du théisme philosophique comme de la doctrine moderne de la double existence, celle qui suppose l'existence d'un objet au-delà de la perception, et dont le Traité fait la critique et la généalogie : «cette hypothèse philosophique ne s'impose primitivement ni à la raison ni à l'imagination », et par conséquent, «le système philosophique tire toute son influence sur l'imagination du système ordinaire»! En cherchant à résoudre les contradictions du sens commun, les philosophes n'ont fait qu'y superposer les leurs. Leur système « est sujet aux mêmes difficultés et, de surcroît, il doit supporter la double absurdité de dénoncer et d'établir à la fois la supposition vulgaire » (TNH, I, IV, II, 296-303). De même peut-on dire du système théiste qu'il ne s'impose primitivement ni à l'imagination du vulgaire, qui ne contemple pas les œuvres de la nature, ni à la raison du vrai philosophe qui les contemple attentivement et n'y voit qu'une compensation universelle de biens et de maux incompatible avec l'idée d'un sage auteur 
souveraine en portant ses attributs à l'infini ${ }^{28}$. Certes, la conclusion de ce développement laisse penser que l'apparition de la religion naturelle ne doit rien à cette dynamique superstitieuse : «Aussi longtemps qu'ils [les hommes] s'en tiennent à la notion d'un être parfait, créateur du monde, ils coïncident par hasard avec les principes de la raison et de la vraie philosophie, bien qu'ils soient menés à cette notion, non par la raison dont ils sont dans une grande mesure incapables, mais par les adulations et les craintes de la superstition la plus vulgaire ${ }^{29}$. Mais il est évident, pour un lecteur avisé de L'histoire naturelle, que cette représentation des choses tient de l'artifice rhétorique, et que l'affirmation d'une coïncidence hasardeuse entre le théisme philosophique et le théisme populaire est une simple concession à l'illusion rétrospective qui fait de la superstition une corruption de la religion vraie et originelle. En réalité, si les hommes ne sont pas menés à la notion philosophique de la divinité par la raison, ce n'est pas seulement parce qu'ils en sont dans une grande mesure incapables, mais c'est aussi et plus fondamentalement parce que la raison elle-même est incapable de les y conduire, et que par conséquent seule la crainte superstitieuse peut le faire.

Les derniers paragraphes de cette sixième section, ainsi que les deux sections suivantes, ont pour objet de confirmer cette naissance du théisme à partir du polythéisme par la persistance de celui-ci dans celui-là. Les relents de polythéisme dans le théisme sont les marques par lesquelles celui-ci, dans son développement même, trahit ses origines. Certes le théisme dont il est question ici est celui des trois grandes religions révélées, et non celui de la religion naturelle, à supposer qu'ils puissent réellement se distinguer l'un de l'autre. Mais on verra comment la dialectique superstitieuse qui oppose l'anthropomorphisme au panégyrique dans le flux et le reflux du théisme et du polythéisme affecte aussi la religion naturelle, et ce jusque dans ses arguments ${ }^{30}$.

Que la religion naturelle soit pleinement l'objet de L'histoire naturelle de la religion, c'est ce que confirme la comparaison systématique entre le théisme et le polythéisme qu'établissent les

de la nature.

28. HNR, VI, 64-65.

29. Ibid. (je souligne).

30. Voir infra, p. 25-27. 
sections IX à XIV de cette dissertation. On dira que les exemples donnés par Hume pour illustrer les inconvénients du théisme sont tous tirés de l'histoire des religions révélées, et principalement de l'histoire du christianisme. Mais la comparaison du théisme et du polythéisme est en réalité indifférente à la question des formes historiques particulières du théisme. Il importe peu que l'on ait cherché à établir celui-ci sur la révélation ou sur la raison, puisque c'est en tant que théisme, c'est-àdire en vertu de son essence même, que celui-ci entraîne la plupart du temps des inconvénients supérieurs à ceux du polythéisme. C'est comme monothéisme, c'est-à-dire comme croyance en une divinité unique qu'il entraîne un exclusivisme intolérant, et par conséquent sa propre division en sectes opposées, portées à la persécution mutuelle (section IX) ; c'est parce qu'il implique la représentation d'un Dieu infiniment supérieur à l'homme que le théisme inspire l'humilité et entrave les vertus civiques ( redoutable et haïssable (section XIII) ; enfin, il suffit que les hommes croient en l'existence d'une divinité (ou de plusieurs) pour qu'ils en viennent à mépriser leurs devoirs envers leurs semblables au profit des devoirs futiles que leur impose leur culte religieux (section XIV). Or, aucune de ces caractéristiques n'est propre au théisme chrétien, qui au contraire les partage toutes avec celui de la religion naturelle.

Il est vrai que dans ces pages Hume prend soin de préciser à chaque fois que c'est lorsque s'y joint la crainte superstitieuse que le théisme entraîne ces effets pernicieux. Mais il est nécessaire que la religion naturelle s'accompagne de superstition à divers degrés, par la conjonction de deux facteurs : sa propre origine superstitieuse et sa vocation à se communiquer à l'ensemble de la société et donc à la multitude ignorante. On a vu plus haut comment la religion des philosophes avait ellemême son origine dans la superstition. Ce qu'il faut montrer à présent, c'est que sa diffusion au sein de la nation entière a pour effet de réactiver ou d'accuser cet élément superstitieux qu'elle renferme toujours de façon plus ou moins latente chez les philosophes eux-mêmes.

Si elle est d'abord une religion de philosophes, la religion naturelle n'en a pas moins pour 
vocation de se communiquer au peuple, et ce pour deux raisons, qui expliquent en même temps qu'elle doive nécessairement dégénérer en superstition.

La première raison est extrinsèque, tenant à la relation que la religion naturelle entretient dans l'histoire avec le christianisme. L'identité entre le christianisme et la religion naturelle n'est pas seulement théorique, au sens où l'on a affaire à un théisme dans les deux cas, en tant qu'on oppose celui-ci au polythéisme. Leur confusion est aussi bien réelle et historique, puisque, certains déistes mis à part, les tenants de la religion naturelle sont presque toujours des chrétiens, voire des prêtres chrétiens $^{31}$. De ce point de vue, la religion naturelle n'est qu'une arme de plus aux mains de ces derniers, qu'ils utiliseront pour asseoir leur pouvoir sur la superstition populaire. Tout porte à croire que Hume devait considérer la religion naturelle comme la forme que prenait au XVIIIème siècle la subordination de la philosophie aux intérêts de la religion, c'est-à-dire aux intérêts temporels du clergé. L'histoire naturelle explique comment le théisme diffusé à grande échelle engendre cette subordination : «là où le théisme constitue le principe fondamental d'une religion populaire, sa doctrine apparaît si conforme à la saine raison, que la philosophie est capable de s'incorporer à un tel système théologique. Et si les autres dogmes de ce système sont contenus dans un livre sacré, tel que le Coran, ou déterminés par une autorité visible, comme celle du pontife romain, les penseurs spéculatifs y étendent leur assentiment et embrassent une théorie qui leur a été inculquée par leur première éducation et qui possède aussi un certain degré de cohérence et d'uniformité. Mais comme il est sûr que toutes ces apparences se révéleront trompeuses, la philosophie se retrouvera bientôt en état d'infériorité sous le joug qui la lie à son nouvel associé. Et au lieu de contrôler chaque principe, sur leur route commune, elle est à chaque pas détournée et mise au service de la superstition $»^{32}$. Certes, le théisme et la philosophie dont il est question dans ce paragraphe sont respectivement le théisme de la religion révélée et la métaphysique «bâtarde » qui sert d'auxiliaire à la théologie et

31. Voir L. Stephen, History of English Thought in the Eighteenth Century, London, Harbinger Book, new. ed. $1962\left(1^{\text {st }}\right.$ ed. 1876), vol. I, p. 72.

32. HNR, XI, 77-78. Voir aussi Enquête sur l'entendement humain, Paris, Le Livre de Poche, 1999, trad. D. Deleule, section XI, p. 239-241 (noté ci-après EEH, suivi de l'indication de la section en chiffres romains et de la page en 
donc au pouvoir des prêtres ${ }^{33}$. Mais en un siècle newtonien, ce sont les apparences d'une philosophie expérimentale que les prêtres devront donner à leur théisme. Si le XVIIème siècle fut marqué par la connivence du christianisme avec la philosophie cartésienne, le XVIIIème siècle se caractérise par l'alliance du christianisme avec la philosophie expérimentale ${ }^{34}$. Telle est l'intrigue des prêtres qu'après avoir fondé la foi sur le scepticisme, lorsque l'humanité était encore ignorante et que l'éducation avait encore un grand poids, ils ont changé de stratégie pour affecter le dogmatisme, d'inspiration cartésienne ou newtonienne, désormais que les lumières se sont répandues et que l'esprit critique a fait reculer l'autorité de l'éducation ${ }^{35}$. La religion naturelle, ce n'est que la religion chrétienne remise au goût du jour, et c'est pourquoi sa diffusion risque d'entraîner des conséquences analogues à celle du christianisme.

Mais le risque de voir la religion naturelle dégénérer en superstition populaire tient aussi à une raison intrinsèque à cette religion, à savoir sa nature contradictoire de philosophie spéculative qui prétend avoir des conséquences pratiques ${ }^{36}$. Cette dernière prétention est clairement affirmée par Cléanthe dans la dernière partie des Dialogues : «La religion a pour office propre de régler le cœur des hommes, d'humaniser leur conduite, de leur insuffler l'esprit de la tempérance, de l'ordre et de l'obéissance ${ }^{37}$. Et rien ne sert d'établir les attributs naturels de la divinité, si ses attributs

chiffres arabes), où l'ami que Hume fait parler donne une explication semblable.

33. EEH, I, 52-53. C'est l'alliance fâcheuse de la métaphysique classique avec la théologie chrétienne qui motive chez Hume la critique d'un certain nombre de thèses classiques dans l'EEH et davantage encore dans le TNH (causalité de Dieu, immatérialité de l'âme, identité personnelle, libre arbitre), auxquels il faut ajouter la dissertation «De l'immortalité de l'âme » destinée à être publiée avec L'histoire naturelle et trois autres dissertations, avant que Hume ne la retire de la publication en 1756 (voir E.C. Mossner, « Hume's Four Dissertations »).

34. J. Butler, auteur de The Analogy of Religion, Natural and Revealed, to the Constitution and Course of Nature (1736), figure dans la liste des philosophes anglais qui, selon Hume, ont commencé à établir la science de l'homme sur la méthode expérimentale (TNH, Introduction, 53, note). Et c'est parce qu'en Butler sont excellemment réunis le théologien anglican et le philosophe expérimental que Hume, d'après E.C. Mossner, en aurait fait la principale cible des Dialogues, sous les traits de Cléanthe (E.C. Mossner, « The Enigma of Hume », Mind, XVL, p. 334-349).

35. DRN, I, 88-89. C'est Philon qui parle.

36. Dans l'œuvre de Locke, qui inspirera les théistes anglais du XVIIIème siècle, la première occurrence d'une tentative de preuve de l'existence de Dieu par la lumière naturelle (qui combine l'argument du premier moteur avec celui du dessein) prend place dans une enquête sur l'obligation morale de l'homme envers lui-même, envers ses semblables et envers Dieu, lequel « a sur nous un commandement juste et inévitable et peut à Son gré nous élever ou nous terrasser, et par le même pouvoir de commandement nous rendre heureux ou misérables » (Essays on the Law of Nature, Oxford, ed. W. von Leyden, Oxford University Press, $4^{\text {th }}$ ed. 1970 ( $1^{\text {st }}$ ed. 1954), IV, p. 155, je traduis). C'est donc pour ses conséquences pratiques, relatives au salut, qu'une preuve de l'existence de Dieu est recherchée.

37. DRN, XII, 216. Dans l'EEH, le dialogue de la section XI, intitulée pour la première édition « Des conséquences pratiques de la religion naturelle », a pour objet d'établir, non que la religion naturelle n'a pas de conséquences 
moraux sont douteux ${ }^{38}$. Or, que l'efficacité pratique de la religion naturelle s'étende à la société entière, cela implique que ses positions théoriques soient aussi largement diffusées. Mais voilà qui entre en contradiction avec la nature spéculative de cette religion, dont les arguments, mais aussi la philosophie naturelle sur laquelle ces arguments prennent appui dans le théisme expérimental, échapperont toujours à la grande majorité des hommes. Par conséquent, toute diffusion de la religion naturelle à l'échelle d'une nation entière aura pour effet de raviver le résidu de superstition qui demeurait dans la religion des philosophes, au détriment ce qu'elle avait de philosophique. La dimension proprement philosophique de la religion naturelle, si elle ne réside pas dans la force de ses arguments, dont les Dialogues montreront toute la faiblesse, est dans l'exercice de cette passion calme qu'est la curiosité, et qui est nécessaire pour que l'homme s'élève à la contemplation des œuvres de la nature et à la considération des causes finales. Si la religion naturelle n'est pas théoriquement rationnelle, sa pratique n'en est pas moins raisonnable. Or l'immense majorité d'une nation ne fait pas de philosophie. Ce qu'il est dès lors possible de diffuser de la religion naturelle au sein d'une société entière, ce ne sont pas ses questions et ses problèmes, initiant une pratique du libre examen, mais seulement ses résultats, que l'homme du commun recevra toujours comme de purs dogmes, c'est-à-dire comme des articles de foi déracinés de leurs fondements rationnels. C'est pourquoi en exprimant des opinions religieuses, ces dogmes ne manqueront pas d'entretenir la superstition du peuple, et d'entraîner tous les effets caractéristiques de la croyance théiste en tant que telle, puisque les dogmes de la religion naturelle une fois coupés des arguments philosophiques

pratiques, mais qu'elle ne devrait pas en avoir, si elle était théoriquement conséquente. C'est ainsi que l'ami de Hume, faisant parler Épicure, commence son discours aux Athéniens : «Il suffit que je puisse prouver, d'après ce raisonnement même [celui du théisme expérimental], que la question est entièrement spéculative, et que, lorsque je nie l'existence d'une Providence et d'un état futur, loin de saper par là les fondements de la société, j'avance des principes qu'ils [les stoïciens, c'est-à-dire les théistes] doivent eux-mêmes, d'après leur propres arguments, s'ils raisonnent de façon conséquente, reconnaître pour solides et satisfaisants » (EEH, XI, 244). Hume, dans sa dernière réplique, confirmera l'intention de son ami, c'est-à-dire la sienne propre en tant qu'auteur : «Vous concluez que les doctrines et raisonnements religieux ne peuvent avoir d'influence sur la vie, parce qu'ils ne doivent pas en avoir, sans aucunement considérer que les hommes ne raisonnent pas de la même manière que vous, mais tirent beaucoup de conséquences de la croyance en une existence divine, et supposent que la Divinité infligera des punitions au vice et accordera des récompenses à la vertu, au-delà de ce qui paraît dans le cours ordinaire de la nature. Que ce raisonnement qu'ils font soit juste ou non, peu importe. Son influence sur leur vie et leur conduite ne peut qu'être toujours la même » (EEH, XI, 260, Hume souligne).

38. DRN, X, 183. 
qui les sous-tendent ne se distinguent plus en rien des dogmes chrétiens. Et cette dégénérescence de la religion naturelle en superstition populaire sera d'autant plus inévitable que la diffusion de cette religion aura lieu au sein d'une société déjà chrétienne, dont le peuple superstitieux ne pourra que recevoir de manière superstitieuse les dogmes théistes.

Mais quand bien même les partisans de la religion naturelle ne seraient pas des prêtres chrétiens, quand bien même le peuple n'aurait jamais connu le christianisme, la religion naturelle n'en aurait pas moins les effets socialement nuisibles de ce dernier, qui tiennent tous à la superstition populaire entretenue par le pouvoir des prêtres. Comme le montre l'essai « Superstition et enthousiasme », avant même que ce pouvoir ne s'appuie sur la superstition ou ne l'entretienne, « la superstition favorise la puissance des prêtres », c'est-à-dire qu'elle la réclame et la fait naître. A l'opposé de l'enthousiaste, le fidèle superstitieux, craignant de s'adresser en personne à la divinité, aura besoin d'un médiateur, le prêtre, ce qui ne manquera pas d'arriver lorsque la divinité présentera les attributs élogieux que lui prête tout théisme. La conséquence en est «qu'on trouvera des prêtres dans presque toutes les sectes religieuses et que leur autorité sera toujours proportionnelle au plus ou moins de superstition qui y règne $»^{39}$. En somme, la religion naturelle retournera d'autant plus à son origine superstitieuse, le théisme populaire, qu'elle se diffusera à plus grande échelle, comme elle l'exige pourtant d'elle-même. Ce sont là toutes les conséquences pratiques, funestes pour la société, auxquelles elle pourra prétendre.

Cette lecture de L'histoire naturelle qui fait de la religion naturelle l'objet ultime de l'enquête causale de cette dissertation sera confirmée s'il est possible de montrer que la superstition est le ressort de l'argumentation de Cléanthe et de Déméa dans les Dialogues.

A cette fin, on peut suggérer de lire les Dialogues de la même façon que L'histoire naturelle,

39. HNR, « Superstition et enthousiasme », 34-35. 
c'est-à-dire à partir de la fin. Le final sceptique de la dissertation invitait à relire celle-ci en y cherchant une histoire naturelle de la religion naturelle elle-même. De façon analogue, la douzième partie des Dialogues, où Cléanthe manifeste ce qui demeure en lui de superstition, invite à relire les Dialogues en y cherchant les principes superstitieux qui commandent l'argumentation de ce personnage, mais aussi de Déméa.

Si Déméa est absent du dernier acte des Dialogues, c'est parce qu'il a quitté subitement ses compagnons à la fin de la onzième partie. Se reconnaissant probablement dans le propos de Philon sur l'intrigue des théologiens - qui consiste à épouser les théories les plus favorables à leur cause en fonction de l'air du temps -, Déméa renonce à poursuivre la discussion, sous l'effet de la passion ${ }^{40}$. Philon reste donc seul avec Cléanthe, c'est-à-dire avec le représentant de la version du théisme qui devait apparaître à Hume comme étant la plus philosophique, sa version expérimentale ${ }^{41}$. Or la manière dont Cléanthe défend la cause de la religion en discutant avec Philon de son utilité pour l'humanité montre que ce philosophe qu'est pourtant Cléanthe ne l'est pas encore tout à fait, en raison de sa conception encore superstitieuse de la religion. Tout le propos de Philon dans cette dernière partie, proche en cela du discours d'Épicure dans l'Enquête, est de montrer que la conclusion légitime du théisme expérimental, à savoir «que la cause ou les causes de l'ordre dans l'univers présentent probablement quelque lointaine analogie avec l'intelligence humaine ${ }^{42}$ réduit le théisme à une opinion purement spéculative, incapable d'avoir la moindre influence sur la conduite humaine. Le corollaire positif de cette thèse est que la morale et la société ont leur fondement en elles-mêmes, et n'ont pas besoin du rempart de la religion, qui au contraire leur est toujours nuisible. Or c'est ce que Cléanthe ne peut supporter d'entendre, lui qui continue de concevoir la vertu comme ayant un mobile extrinsèque, dans l'espoir d'une récompense et la crainte d'un châtiment, en ajournant cette rétribution à la vie future, comme l'exprime chacune de ses répliques à Philon. Or l'espoir et plus encore la crainte sont les passions qui, jointes à l'ignorance,

40. DRN, XI, 204-205.

41. Voir supra, p. 17. 
produisent la superstition... Mais puisque Cléanthe et Déméa ne sont pas des ignorants mais des philosophes, leurs dispositions superstitieuses doivent avoir affecté leurs arguments au cours des Dialogues. Les onze premières parties de l'ouvrage confirment-elles cette hypothèse ?

La discussion des arguments en faveur de la croyance théiste ne commençant qu'avec la deuxième partie des Dialogues, la première partie confirme d'abord les dispositions superstitieuses des interlocuteurs de Philon ${ }^{43}$. De ce point de vue, il est significatif que Philon représente ces dispositions dans une histoire - sans que ses compagnons ne se reconnaissent dans cette histoire qui est celle de l'intrigue des prêtres. La stratégie sceptique que Déméa met au service de son mysticisme et «la tournure philosophique » de Cléanthe ${ }^{44}$ représentent de manière contemporaine les stratégies successivement adoptées par les prêtres au cours de l'histoire, prêchant le scepticisme à une humanité encore ignorante, affectant le dogmatisme dès lors que les lumières se furent propagées parmi les hommes. Cléanthe approuve immédiatement la stratégie dogmatique : la valeur de vérité des principes spéculatifs est fonction de leur aptitude à servir les intérêts de la religion dans sa lutte contre les athées, les libertins et les libres penseurs ${ }^{45}$. Quant à Déméa, son programme éducatif consiste bien à inculquer le scepticisme pour mieux faire accepter les mystères de la religion $^{46}$. Vue sous cet angle, la religion naturelle apparaît entièrement apologétique.

L'entrée en matière, dans la seconde partie, représente pourtant les arguments théistes comme pourvus d'une autonomie philosophique et d'une signification théorique certaines, sans quoi la critique sceptique de Philon n'aurait aucune prise. Pour s'en apercevoir, il suffit de convoquer le premier argument de chacun des deux théistes, et de voir la réponse qu'ils entraînent de la part de Philon. Lorsque Déméa, citant Malebranche ${ }^{47}$ avance l'argument de l'équivocité pour affirmer la nature mystérieuse de Dieu, Philon s'accorde avec lui pour dire que nous n'avons aucune idée des

42. DRN, XII, 226.

43. La discussion des arguments en faveur de la croyance théiste (parties II à XI) est donc encadrée par deux entretiens révélant les dispositions non philosophiques qui président à ces arguments (parties I et XII).

44. DRN, «Pamphile à Hermippe », 74.

45. DRN, I, 88-90.

46. DRN, I, 75-76. 
attributs de Dieu, par où il laisse entendre clairement que l'existence de Dieu ne nous est pas davantage connue, s'il est vrai que nous ne pouvons croire en l'existence de ce dont nous n'avons pas même l'idée ${ }^{48}$. De même lorsque Cléanthe formule pour la première fois l'argument de l'analogie, la critique de Philon s'appuie sur le principe de la proportion directe entre le degré de ressemblance des termes analogues et la force (ou la faiblesse) de l'analogie ${ }^{49}$. La teneur authentiquement philosophique des arguments de Déméa et de Cléanthe est attestée par la nécessité où se trouve la critique sceptique de faire intervenir des principes de cette partie de la science de l'homme qu'est la logique.

Mais puisque les opinions théistes sont des erreurs, elles doivent avoir une origine, à défaut d'avoir quelque fondement. Or cette origine est la superstition, dans sa tendance louangeuse chez Déméa, et dans sa tendance contraire, idolâtre, chez Cléanthe. Les arguments rappelés plus haut donnent le ton de la posture qu'adopteront chacun des compagnons de Philon tout au long des Dialogues. Le mysticisme de Déméa ne fait que répéter dans un langage philosophique le panégyrique frénétique qui dans L'histoire naturelle caractérisait l'acte de naissance du théisme à partir du polythéisme : «parce qu'ils [les hommes] imaginent que, comme eux, [le dieu souverain] aime la louange, ils ne lui épargneront aucun éloge, aucune exagération dans leurs suppliques. Plus ils sont en proie à la crainte et à la détresse et plus ils inventent de nouvelles sortes d'adulations ; et celui qui dépasse ses prédécesseurs dans l'art de gonfler les titres divins est assuré d'être lui-même surpassé par ses successeurs, qui découvriront des épithètes élogieuses plus neuves et plus pompeuses. Ainsi progresse-t-on jusqu'à ce qu'on arrive ainsi à l'infini lui-même au-delà duquel il n'y a plus de progrès possible ; et tout est bien si, dans leurs tentatives pour aller plus loin et pour représenter une simplicité magnifique, les hommes ne se jettent pas dans des mystères inexplicables et ne détruisent pas la nature intelligente de leur divinité, sur laquelle seule un culte ou une

47. Recherche de la vérité, Livre III, Partie II, ch. IX, § IV.

48. L'idée d'existence n'est pas distincte de l'idée de ce que nous concevons comme existant, et donc réciproquement, si nous ne concevons rien, nous ne concevons rien d'existant. Voir TNH, I, II, VI, 122-123. 49. Voir TNH, I, III, XII, 214-215. 
adoration rationnelle peut être fondée ${ }^{50}$. Les propos par lesquels Déméa, avant même de citer Malebranche, commence par justifier la nature mystérieuse et incompréhensible de Dieu font puissamment écho, par le champ lexical du sacré qu'ils mettent en place, au passage précédent de L'histoire naturelle : «Créatures finies, faibles et aveugles, nous devons nous humilier devant son auguste Présence et, conscients de notre fragilité, adorer en silence ses infinies perfections que l'œil n'a point vues, que l'oreille n'a pas entendues, et qu'il n'est pas entré dans le cœur de l'homme de concevoir. Elles sont voilées par une profonde nuée à la curiosité humaine ; c'est une profanation de tenter de pénétrer ces obscurités sacrées. Et proche de l'impiété de nier l'existence de Dieu, vient l'audace de chercher à scruter sa nature et son essence, ses décrets et ses attributs » ${ }^{51}$. Quant à l'anthropomorphisme de Cléanthe, son origine est encore plus lointaine et populaire, puisque la tendance des hommes à imaginer « des visages humains dans la lune, des armées dans les nuages », est caractéristique du polythéisme, qui fut la religion primitive de l'humanité ${ }^{52}$. Le premier raisonnement de Cléanthe présente pourtant toutes les apparences d'un raisonnement philosophique en bonne et due forme, puisqu'il suit «toutes les règles de l'analogie ». Étant donné que le monde ressemble à une machine, il doit avoir pour auteur un être semblable à l'intelligence humaine. A quoi Philon objecte que le monde ne ressemble que de très loin à une maison, et que l'analogie est par conséquent trop faible pour autoriser autre chose qu'une simple conjecture. L'erreur de Cléanthe ne semble pas résider, à première vue, dans un vice de forme affectant son raisonnement, mais dans une observation erronée de la relation de ressemblance entre les effets. Négligeant les différences entre les produits de l'art humain et le monde, Cléanthe est conduit à reporter cet abus de ressemblance sur les causes, concluant à une similitude entre l'intelligence humaine et l'auteur du monde. Or d'où vient son erreur d'observation? L'explication la plus prochaine est dans le penchant de l'imagination à suivre la facilité des transitions qu'elle opère d'une idée à l'autre et qui est responsable des règles générales et de cette sorte de probabilité non philosophique qu'est le 
préjugé $^{53}$. Mais l'activité de l'imagination n'est jamais autonome, elle est toujours motivée par quelque passion ou inclination. Dans le cas de Cléanthe, il peut s'agir de la curiosité, mais d'une curiosité précipitée par un reste de superstition idolâtre qui le conduit à chercher avant tout à justifier sa croyance en l'existence d'une puissance invisible, et à le faire en suivant à son insu cette «tendance universelle [des hommes] à concevoir tous les êtres à leur ressemblance » ${ }^{54}$. Autrement dit, il y a bien un vice de forme dans le raisonnement de Cléanthe, qui réside dans une pétition de principe d'un genre particulier : c'est parce qu'il suppose inconsciemment que la divinité doit ressembler en quelque manière à l'intelligence humaine que Cléanthe croit observer une ressemblance entre le monde et les artefacts. L'inférence du théisme expérimental est donc préjugée, en vertu de la persistance de l'idolâtrie dans l'esprit scientifique, de même que la doctrine de l'équivocité avancée par Déméa manifestait la persistance du panégyrique.

Mais en réalité, l'opposition de l'idolâtrie et du panégyrique ne se superpose pas exactement à l'opposition entre Cléanthe et Déméa, puisque le conflit entre les deux théistes révèle aussi bien leurs points communs. Le mysticisme de Déméa est aussi bien anthropomorphique en son principe, qui est de s'imaginer que la divinité, comme les hommes, aime la louange. Inversement, l'anthropomorphisme de Cléanthe est aussi bien louangeur, dans la mesure où il ne détermine la nature de Dieu qu'en portant les qualités humaines à l'infini. Conduit par son anthropomorphisme à affirmer que «l'Auteur de la nature est en quelque manière semblable à l'esprit de l'homme », Cléanthe est néanmoins porté par le panégyrique à concéder que Dieu est « doué de facultés beaucoup plus vastes ». Il est vrai que cette concession semble reposer sur l'observation d'une différence entre le monde et les produits de l'art humain, puisque l'adaptation des moyens aux fins dans la nature «dépass[e] de beaucoup » tout ce qu'on peut observer d'analogue dans les objets artificiels, et qu'il convient par conséquent de proportionner la différence entre les causes à la différence entre les effets. Mais si Cléanthe était mû par une curiosité pure de toute superstition, il 
aurait dû, en raison de cette dernière différence, reconnaître immédiatement la faiblesse proportionnelle de l'analogie que lui objectera Philon, au lieu de porter à l'infini les attributs divins. Mais c'est ce qu'une crainte superstitieuse l'empêchera de faire. Dans les arguments théistes, le panégyrique se mêle toujours, et contradictoirement, à l'anthropomorphisme.

Envisagés de ce point de vue, les personnages des Dialogues acquièrent une valeur allégorique. Il est vrai qu'on peut voir en eux l'incarnation de personnes réelles ou de positions philosophiques particulières et historiques ${ }^{55}$. Mais si l'on s'en tient là, on ne verra dans les Dialogues que la critique ou la censure d'opinions particulières, alors qu'ils ont en réalité une portée positive pour la science de l'homme, au même titre que l'enquête causale de L'histoire naturelle. Cléanthe et Déméa ne représentent pas seulement Clarke et Butler ou leurs écoles, mais figurent de manière allégorique des tendances naturelles de la raison humaine lorsqu'elle porte ses vues au-delà de l'expérience, tendances qui obéissent à des principes généraux de la nature humaine, ceux de la superstition, et que L'histoire naturelle a révélées. Polythéisme et monothéisme, idolâtrie et panégyrique, anthropomorphisme et mysticisme ne sont pas tant des phénomènes réels que des modèles théoriques, des cas limites ou des épures qui ensemble offrent une grille de lecture des religions historiques. Ils ne se trouvent jamais nulle part à l'état pur, mais toujours mélangés, formant des mixtes que L'histoire naturelle de la religion permet d'analyser ${ }^{56}$.

La dialectique insoluble entre les positions antithétiques de Cléanthe et de Déméa, qui ouvre la quatrième partie des Dialogues, met en scène la dialectique infernale de la superstition, lorsque s'opposent ses deux tendances contradictoires, l'anthropomorphisme et la louange. Pour s'en apercevoir, il faut faire retour à la section VIII de L'histoire naturelle qui décrivait ce processus pour rendre compte du flux et du reflux du polythéisme et du théisme : «Du fait de la débilité de

54. HNR, III, 48.

55. Voir E.C. Mossner, «The Enigma of Hume », qui en assimilant Cléanthe à Joseph Butler et Déméa à Samuel Clarke, complète les analyses de N. Kemp Smith qui, dans son édition des Dialogues (Oxford, 1935), assimilait Philon à Hume.

56. Sur le caractère sciemment hypothétique et conjectural des histoires naturelles appliquées à l'homme dans la philosophie britannique des XVIIème et XVIIIème siècles, voir P. Carrive, La pensée politique anglaise de Hooker à 
leur faible entendement, les hommes ne peuvent se contenter de concevoir leur divinité sous la forme d'un pur esprit et d'une intelligence parfaite ; et cependant leurs terreurs naturelles les empêchent de lui conférer la moindre ombre de limitation ou d'imperfection. Ils flottent entre ces sentiments opposés. La même faiblesse les tire toujours vers le bas : d'une divinité omnipotente et spirituelle vers une divinité corporelle et limitée et de là à une statue ou à une représentation visible. Le même effort d'élévation les pousse toujours vers le haut : d'une statue ou d'une image matérielle vers une puissance invisible et de là vers un dieu infiniment parfait, créateur et souverain de l'univers ${ }^{57}$. Or dans les Dialogues, cette dialectique de la superstition populaire se reproduit au sein même du théisme, et du théisme philosophique. Quand les analogies de Cléanthe tirent la croyance théiste vers le bas, la théologie négative de Déméa s'efforce de la tirer vers le haut. Et ce qui atteste que leur opposition repose sur des principes superstitieux, c'est qu'ils s'accusent mutuellement de sacrilège. De son côté, Cléanthe comprend bien qu'en vidant l'idée de Dieu de tout contenu représentable, on rend douteuse jusqu'à l'existence de Dieu : «Est-ce le nom, privé de toute signification, qui est d'une si considérable importance ? Ou en quoi, vous autres mystiques, qui affirmez l'incompréhensibilité absolue de la Divinité, différez-vous des sceptiques et des athées, qui prétendent que la cause première de toutes choses est inconnue et inintelligible ? ${ }^{58}$. A quoi Déméa rétorque, en reprochant à son interlocuteur une bigoterie dont il n'est pas exempt : «Qui aurait pu imaginer, répondit Déméa, que Cléanthe, le calme, le philosophique Cléanthe, tenterait de réfuter ses adversaires en leur attachant un sobriquet, et, à l'image des vulgaires bigots et inquisiteurs de notre temps, aurait recours à l'invective et à la déclamation plutôt qu'au raisonnement ? Ou ne s'aperçoit-il pas que ses procédés sont aisés à retourner et que l'appellation d'anthropomorphite est aussi odieuse et implique d'aussi dangereuses conséquences que l'épithète de mystique dont il nous a honorés ? ${ }^{59}$. Dieu ne peut être dit posséder un esprit semblable à celui de l'homme, qui se

Hume, Paris, PUF, 1994, p. 339 sq.

57. HNR, VIII, 70-71.

58. DRN, IV, 117.

59. Ibid., 118. 
caractérise par un changement continuel de ses perceptions. C'est ainsi que le ressort superstitieux de l'opposition entre les deux personnages empêche la discussion de jamais se reposer en trouvant la juste mesure de ce que l'on peut déterminer précisément de la nature divine, juste mesure que la patience spéculative de Philon s'efforcera d'approcher dans la suite des Dialogues. L'anthropomorphisme de Cléanthe et le mysticisme de Déméa, cherchant à se compenser réciproquement, ne donnent naissance qu'à deux excès opposés, qui constituent deux erreurs, l'abus de la relation de ressemblance dans un cas, la perte de sens du discours dans l'autre.

Mais il n'est pas jusqu'aux variations que Philon, dans les parties V à VIII des Dialogues, fait subir à l'hypothèse religieuse qui ne fassent écho à L'histoire naturelle ${ }^{60}$. Certes ces suppositions, motivées par un authentique désir de connaître exempt de toute superstition, constituent autant d'efforts pour maintenir l'inférence au plus près de la réalité empirique du monde. Mais leur équivalence ou leur égale possibilité, que Philon reconnaît, repose sur une absence de fondement analogue à celle qui autorisait les variations de la mythologie païenne ${ }^{61}$. C'est pourquoi Cléanthe peut présenter les hypothèses de Philon comme des «fantaisies » qui «peuvent nous déconcerter, mais jamais nous convaincre $»^{62}$. Mais il ne peut en aller autrement dans les questions religieuses : «Ce que vous attribuez à la fertilité de mon invention, répondit Philon, est entièrement dû à la nature du sujet. Dans les sujets qui sont adaptés à l'étroit registre de la raison humaine, il n'y a d'ordinaire qu'une seule façon de se déterminer, qui emporte avec elle probabilité et conviction ; et à

60. Selon ces variations, le monde peut être : le produit d'une collaboration de plusieurs démiurges (V, 130-132), ou du travail bâclé d'une divinité médiocre (V, 133) ; un grand animal mis en action par « l'âme du monde » des anciens (VI, 136-138), une matière à laquelle un principe d'ordre inhérent donne ses formes en de perpétuelles révolutions (VI, 141 142), un globe gouverné par 30.000 divinités, comme dans la théologie païenne d'après Hésiode (VI, 142-143) ; une sorte de végétal ou d'animal dont la cause est semblable à la végétation ou à la génération (hypothèse que Philon fait varier elle-même tout au long de la septième partie) ; une quantité finie d'atomes reproduisant éternellement un nombre fini de configurations (VIII, 155-157), une matière mue par une force inhérente et aveugle capable de conserver les formes de la matière à travers un changement continuel de ses parties (VIII, 157-160). Il est significatif que la plupart de ces hypothèses soient inspirées de la Grèce ancienne, c'est-à-dire d'une époque encore largement polythéiste.

61. «En bref, tout le système mythologique est si naturel, que dans l'immense variété des planètes et des mondes contenus dans l'univers, il y a de grandes chances pour que ce système soit réellement mis en œuvre quelque part. La principale objection qu'on y peut faire, quant à notre planète, est qu'aucune raison ni aucune autorité ne le rendent certain. La tradition ancienne, mise en avant par les prêtres et les théologiens païens, n'est qu'un faible fondement; de plus, elle a transmis un si grand nombre de versions contradictoires, toutes soutenues d'une égale autorité, qu'il est devenue impossible d'accorder sa préférence à l'une d'entre elles » (HNR, XI, 77).

62. DRN, VII, 153. 
un homme de jugement sain, toutes les autres suppositions, à l'exception de celle-ci, paraissent entièrement absurdes et chimériques. Mais dans les questions comme la présente, cent vues contradictoires conservent une sorte d'analogie imparfaite ; et l'invention a ici pleine latitude pour s'exercer. Sans grand effort de pensée, je crois pouvoir dans l'instant proposer d'autres systèmes de cosmogonie, qui auraient quelque faible apparence de vérité ; bien qu'il y ait une chance sur mille, sur un million, pour que le vôtre ou l'un des miens soit le vrai système ${ }^{63}$. Or, c'est en affirmant la grande variété de la croyance religieuse, qui «n'a produit aucune uniformité dans les idées qu'elle a fait naître », que l'Introduction de L'histoire naturelle justifiait le projet d'une enquête sur l'origine de la religion dans la nature humaine. C'est parce que les variations de la croyance religieuse dépendent de «divers accidents et de diverses causes » qu'il est possible d'en faire l'histoire naturelle ${ }^{64}$. Or si les croyances populaires naissent du concours de la crainte des événements du monde naturel et de l'ignorance de leurs causes, les croyances théistes naissent de la rencontre de cette superstition avec la philosophie naturelle au sein d'une humanité plus civilisée. La religion naturelle, ce n'est que la superstition populaire enrichie et raffinée par l'apport de la philosophie naturelle ${ }^{65}$.

En passant de l'examen des attributs naturels (parties II à IX) à celui des attributs moraux de la divinité (parties $\mathrm{X}$ et XI), la discussion ne fait que confirmer le mélange superstitieux de louange et d'anthropomorphisme qui affecte les arguments théistes. L'alliance provisoire entre Philon et Déméa, qui ouvre la dixième partie des Dialogues, repose sur un malentendu (mais il n'y a guère que Déméa qui entende mal) dont la fin de la onzième partie donnera la clé de compréhension, provoquant le départ de Déméa. Si ce dernier s'accorde tout d'abord avec Philon pour brosser le tableau pessimiste du monde, ce n'est pas en vertu d'une observation scrupuleuse et non prévenue du mal physique et moral qui pèse sur l'existence humaine et qui justifie le pessimisme de Philon.

63. DRN, VIII, 155.

64. HNR, Introduction, 39-40.

65. Philon : «Philosopher sur de tels sujets revient pour l'essentiel à raisonner sur la vie commune ; et nous pouvons seulement attendre plus de stabilité, sinon plus de vérité, de notre philosophie, par suite de sa manière plus exacte et 
Le pessimisme de Déméa n'a rien de philosophique, il est entièrement commandé par la stratégie que Philon démasque dans ce qu'il appelle « l'intrigue des prêtres », qui « savent changer leur style selon les époques », inculquant le scepticisme et le pessimisme à une humanité inculte et docile, affectant le dogmatisme et l'optimisme au sein d'une humanité éclairée ${ }^{66}$. La réaction de Cléanthe le prouve. Voyant où conduit le pessimisme de ses compagnons, il s'empresse de leur opposer un optimisme ad hoc : «La seule méthode pour étayer la divine bienveillance (et c'est celle que j'adopte bien volontiers) est de nier absolument la misère et la méchanceté de l'homme. Vos représentations sont exagérées ; vos vues mélancoliques sont, pour la plupart, fictives ; vos inférences sont contraires au fait et à l'expérience. La santé est plus commune que la maladie ; le plaisir que la douleur ; le bonheur que la misère ; et pour une souffrance endurée, nous obtenons, au bout du compte, cent jouissances ${ }^{67}$. Parce qu'il a a priori pour objectif d'établir la nature bienveillante de la divinité, Cléanthe est conduit à fermer les yeux sur la proportion du mal dans le monde, réitérant ici la pétition de principe et l'erreur d'observation par lesquelles il avait posé une grande ressemblance entre le monde et les machines fabriquées par l'homme. Si la religion naturelle se distingue de la superstition populaire par la connaissance de la nature qu'elle présuppose, il n'empêche qu'elle comporte encore un certain degré d'ignorance de la réalité du monde. Mais tandis que dans la superstition populaire l'ignorance produit d'abord la crainte, dans la religion naturelle c'est l'inverse. C'est un reste de crainte superstitieuse qui produit l'ignorance, en conduisant le théiste à voir dans la nature ce qui ne s'y trouve pas, ou à ne pas voir ce qui s'y trouve. L'aveuglement de Cléanthe est ici à son comble, puisque pour s'apercevoir que les maux sont en plus grand nombre que les biens, il n'est pas nécessaire de posséder une grande connaissance en philosophie naturelle. La vie courante suffit à cela ${ }^{68}$ !

La onzième partie met encore davantage en évidence la pétition de principe qui commande

plus scrupuleuse de procéder » (DRN, I, 81-82).

66. DRN, XI, 204.

67. DRN, X, 184.

68. Ibid., 187. 
l'optimisme de Cléanthe. Philon vient de montrer, à la fin de la partie précédente, que même si l'on accorde à Cléanthe son optimisme, on ne peut en inférer le caractère infini des attributs de la divinité, puisque l'existence du moindre mal, si l'on proportionne la cause à l'effet, conduit à limiter d'autant ces attributs. Dans sa réponse, qui ouvre donc la onzième partie, Cléanthe, suivant la voie prudente ouverte par Philon, commence par rejeter la méthode louangeuse par laquelle les théologiens de la trempe de Déméa portent à l'infini les attributs divins. Mais la suite de son propos montre qu'il adopte sans s'en apercevoir une méthode identique, associée à sa méthode analogique habituelle. Concédant à la prudence de Philon que l'analogie ne conduit à inférer qu'un dieu fini, il avance un argument de théodicée : «Mais si nous supposons l'Auteur de la nature doué d'une perfection finie, quoique passant de loin l'humanité, alors une explication satisfaisante peut être donné du mal naturel et moral et tout phénomène adverse se voit justifié et éclairci. Un moindre mal peut alors être choisi, afin d'en éviter un plus grand ; des inconvénients acceptés en vue d'atteindre une fin désirable ; et en un mot, la bienveillance, réglée par la sagesse et limitée par la nécessité, peut produire un monde juste comme celui-ci ${ }^{69}$. En démasquant le vice de raisonnement, la réponse de Philon révèle le préjugé superstitieux qui le commande. Si Cléanthe suivait fidèlement sa méthode expérimentale, qui consiste à partir des effets pour remonter aux causes, il ne pourrait même pas inférer l'existence d'un auteur très sage, très bon et très puissant, en raison de la trop grande proportion de mal dans le monde présent. Si donc il croit pouvoir concilier la supposition d'un tel auteur avec l'existence du mal, c'est parce qu'il a commencé par poser a priori l'existence de cet auteur, au lieu de l'inférer à partir du monde, et que, redescendant de la cause à l'effet, il améliore celui-ci pour le rendre conforme à sa cause. D'où la question embarrassante que lui pose Philon : «le monde, considéré en général, et tel qu'il nous apparaît dans cette vie, est-il différent de ce qu'un homme, ou un être limité comparable, attendrait à l'avance d'une divinité très puissante, très sage et très bienveillante ? Il faut un étrange préjugé pour affirmer le contraire ${ }^{70}$. C'est 
pourtant ce qu'affirmait Cléanthe en réduisant la proportion du mal, pour sauver les apparences, mais dans le but premier de sauver la croyance en un dieu très bon, très sage et très puissant ${ }^{71}$. Ici encore, le panégyrique superstitieux conduit le théiste à poser en principe ce qui est en question ${ }^{72}$.

Ce que permet le rapprochement entre L'histoire naturelle et les Dialogues, c'est de saisir la spécificité du fait religieux, et ce qui le distingue de la philosophie. Si l'on remonte à l'origine de la religion pour rendre compte de toutes ses formes particulières, on découvre qu'il n'y a pas deux sortes de religion, celle du peuple et celle des philosophes, mais une seule, qui a pour nom la superstition, en tant que Hume l'oppose à la philosophie ${ }^{73}$. Si l'on peut parler de religion au singulier, et s'il y a bien une philosophie unifiée de la religion chez Hume, c'est parce que la religion n'a lieu qu'en l'absence de philosophie, lorsque l'homme ne fait pas encore de philosophie, et c'est le cas des religions positives dans leur état primitif, ou dès lors qu'il cesse de philosopher ou ne philosophe encore pas tout à fait, et c'est le cas de la théologie révélée, de la métaphysique «bâtarde », mais aussi de la religion naturelle. La cause principale de la superstition populaire, on le sait, est l'ignorance de la nature, lorsque la philosophie naturelle fait défaut. Ce qui est moins évident, c'est que la religion naturelle est encore une superstition, qui tient à un défaut de connaissance, non pas tant de la nature extérieure que de la nature humaine elle-même. Si la

\footnotetext{
71. L'argument a priori avancé par Déméa dans la neuvième partie obéit à un impératif apologétique analogue : puisqu'il est toujours possible de concevoir un être comme non existant, c'est seulement la nécessité de maintenir la croyance en la Divinité qui peut conduire l'esprit à concevoir celle-ci comme un être nécessairement existant.

72. La critique sceptique et le préjugé superstitieux qu'elle dénonce sont les mêmes lorsque, de la justification de l'état du monde présent, on passe à la doctrine de l'état futur, comme le montre l'ami de Hume dans le dialogue de l'EEH : «Vous persistez à imaginez que, si nous accordons cette existence divine, pour laquelle vous combattez si ardemment, vous pouvez sans crainte en inférer des conséquences, et ajouter quelque chose à l'ordre de la nature, tel qu'on le voit être par expérience, en arguant des attributs que vous assignez à vos dieux. Mais vous ne semblez pas vous souvenir que tous vos raisonnements sur ce sujet ne peuvent être tirés que des effets aux causes, et que tout argument déduit des causes aux effets doit être nécessairement un grossier sophisme, puisqu'il vous est impossible de rien savoir de la cause, que ce que vous avez auparavant, non pas inféré, mais découvert en entier dans l'effet » (EEH, XI, 251-252). C'est que la supposition d'une divinité parfaitement juste et toute-puissante «sent plus la flatterie et le panégyrique que le raisonnement juste et la saine philosophie » (Ibid., 259).

73. La conclusion du Livre I du TNH pose clairement l'alternative concernant le guide de la vie humaine : la philosophie est préférable à la superstition, parce que «D'une manière générale, les erreurs, en religion, sont dangereuses ; en philosophie, elles ne sont que ridicules » (TNH, I, IV, VII, 364-365).
} 
philosophie naturelle n'a pas suffi à éradiquer la superstition chez les philosophes, c'est parce qu'ils ont jusqu'ici négligé cette capitale de la philosophie qu'est la nature humaine ${ }^{74}$. Or l'ignorance qui maintient les théistes dans la superstition n'est pas seulement celle de la nature de l'entendement humain, qui les conduit à des paralogismes et des hypothèses arbitraires. C'est aussi et plus fondamentalement celle des principes naturels de la superstition tels qu'ils opèrent à leur insu au sein même de leurs spéculations philosophiques.

Ainsi éclairés par L'histoire naturelle de la religion, qui permet d'exhumer les préjugés superstitieux enfouis dans la conscience théiste, les Dialogues sur la religion naturelle appartiennent de plein droit à la science de l'homme en sa dimension positive, et non seulement critique. La nécessité d'une critique de la religion naturelle ne s'explique, et la critique elle-même ne se comprend pleinement, que si l'on envisage d'abord la religion naturelle du point de vue causal ou naturaliste de L'histoire naturelle. Autrement dit, la critique trouve son sens dans la généalogie. C'est parce que la religion naturelle manifeste la persistance de la superstition dans la philosophie elle-même, ou plus exactement la forme que cette persistance prend de manière privilégiée au XVIIIème siècle, qu'il est nécessaire d'épurer de manière critique ses arguments des préjugés religieux qui les contaminent. L'opposition, qui est aussi bien une alliance, entre la tendance louangeuse et la tendance anthropomorphiste, et qui pousse la raison philosophique à dépasser les limites de l'expérience, est le grand obstacle épistémologique qui empêche le siècle des lumières d'être purement philosophique, et de chercher le fondement de la morale et de la société dans la seule nature humaine. Enfin, si la religion est d'autant plus dangereuse qu'elle revêt mieux les apparences de la philosophie, on comprend que les Dialogues sur la religion naturelle privilégient la critique de la version expérimentale du théisme.

74. TNH, Introduction, 34. 
